\title{
Suppression laws for multiparticle interference in Sylvester interferometers
}

\author{
Andrea Crespi* \\ Istituto di Fotonica e Nanotecnologie, Consiglio Nazionale delle Ricerche, Piazza Leonardo da Vinci 32, 20133 Milano, Italy
}

(Received 3 December 2014; published 8 January 2015)

\begin{abstract}
Quantum interference of correlated particles is a fundamental quantum phenomenon which carries signatures of the statistics properties of the particles, such as bunching or antibunching. In the presence of particular symmetries, interference effects take place with high visibility, one of the simplest cases being the suppression of coincident detection in the Hong-Ou-Mandel effect. Tichy et al., [Phys. Rev. Lett. 104, 220405 (2010)] recently demonstrated a simple sufficient criterion for the suppression of output events in the more general case of Fourier multiport beam splitters. Here we study the case in which $2^{q}$ particles (either bosonic or fermionic) are injected simultaneously in different ports of a Sylvester interferometer with $2^{p} \geqslant 2^{q}$ modes. In particular, we prove a necessary and sufficient criterion for a significant fraction of output states to be suppressed, for specific input configurations. This may find application in assessing the indistinguishability of multiple single-photon sources and in the validation of boson sampling machines.
\end{abstract}

PACS number(s): 42.50.Ct, 03.67.Lx, 42.50.Ex, 05.30.Jp

\section{INTRODUCTION}

Multiparticle quantum interference arises when several indistinguishable particles have a nonvanishing probability amplitude of being found at the same site or spatial coordinate. The algebraic sum of all these probability amplitudes may lead to strong enhancement (constructive interference) or suppression (destructive interference) of the detection probability of the different possible collective states. In that, it is a pure and typical quantum phenomenon, which is worth being investigated both from a fundamental perspective and for its quantum information implications.

Qualitatively different behaviors may be observed in general, depending on the bosonic or fermionic nature of the particles. The antisymmetrization requirements [1] for fermionic wave functions lead to a vanishing probability of finding more than one particle on the same site (the Pauli principle). Bosons, in contrast, show a marked tendency to bunch together, with increased probability to coalesce on the same site [2,3] or to cluster in nearby sites (bosonic clouding [4]).

However, when particles evolve following Hamiltonians with specific symmetries, particular fine-grained distributions can be observed with enhanced interference peaks and dips. The simplest case is the Hong-Ou-Mandel (HOM) effect when two particles impinge on distinct ports of a balanced beam splitter: quantum interference suppresses the coincident output (one particle per each output) in the case of bosons and the single-port output (both particles in either output) in the case of fermions. In the multiparticle case, a sort of generalized HOM effect occurs for symmetric multiport beam splitters [5-8]. In particular, Tichy et al. [7] showed that for a particular class of multiports, namely, Bell or Fourier multiports, and input states with cyclic symmetry, a full suppression of most of the output combinations is observed; a simple analytical law gives a sufficient criterion for such suppression.

From a computational point of view, calculating the output distribution of a number of indistinguishable bosons is a

\footnotetext{
*andrea.crespi@polimi.it
}

mathematically hard problem, in that it cannot be performed efficiently on conventional (classical) computers. In fact, it relies on the calculation of permanents of matrices, for which an efficient classical algorithm is lacking. The realization of such difficulty has led to the proposal [9] of boson sampling devices as experimentally accessible platforms that could perform some task hard to simulate with classical resources. The specialized task of such quantum devices is to physically implement and sample the distribution of $n$ bosons undergoing a certain unitary evolution. The computational difficulty of a classical simulation of such a process (i.e., a classical sampling of such a distribution) increases exponentially with $n$, rapidly becoming infeasible. First proof-of-principle experiments with photons have been reported very recently [4,10-14]; while not having demonstrated yet a true quantum supremacy, these experiments have pointed out that such a demonstration may not be so far from being reached.

A future many-modes boson sampling experiment will likely require the implementation of an arbitrary unitary matrix, through a possibly reconfigurable [15] linear interferometer $[13,16]$. If the output is hard to predict classically, the certification of the correct operation of such device may be not trivial also: in fact, several solutions to this problem have been debated [4,14,17-19]. The use of particular symmetric unitaries that show rich but easily predictable multiphoton distributions has been also proposed [18] as a convenient way to assess the performance both of a multiphoton source as well as of the reconfigurable device itself. In the same way that the two-photon HOM effect on balanced beam splitters has long been used as a diagnostic instrument for measuring two-photon indistinguishability, suppression laws for multiport interferometers could provide a suitable means to simultaneously test the quality of a multiphoton source and of a multimode reconfigurable device for boson sampling experiments. Of course, these could be adopted to assess the performance of a multiphoton source also outside of the boson sampling context.

It has to be noted that the existence of a sharp suppression law directly comes from the symmetry characteristics of the matrix. While it has been conjectured that other suppression laws could exist for other classes of symmetric unitaries, only 
the class of Fourier matrices has been investigated extensively up to now $[7,20,21]$.

In this work we address the study of interferometers implementing $m=2^{p}$-modes Sylvester matrices and prove a necessary and sufficient criterion for the suppression of most output combinations, for certain input states of $n=2^{q} \leqslant m$ particles, either bosonic or fermionic. We further discuss possible applications for assessing the indistinguishability of multiple single-photon sources.

In Sec. II we recall some basic concepts about the evolution of multiparticle Fock states through linear unitary processes and about the definition of Sylvester matrices. In Sec. III we give a comprehensive characterization of the output distributions in the two-particle case, while in Secs. IV and V we prove the output suppression criteria for the cases of multiple bosons and multiple fermions, respectively. These sections are mainly organized as a list of propositions regarding mathematical properties of certain matrices and their consequences on the calculation of the multiparticle output distributions. Finally, we discuss in Sec. VI the consequences and possible applications of the suppression criteria proved in the preceding sections, with particular regard to experiments with photons.

\section{PRELIMINARY CONCEPTS}

\section{A. Multiparticle interference in linear interferometers}

A generic Fock state $|T\rangle$ of $n$ particles on $m$ modes can be written as $|T\rangle=\left(\prod_{i=1}^{n} a_{t_{i}}^{\dagger}\right)|0\rangle$, where $a_{t_{i}}^{\dagger}$ is the creation operator on the mode $t_{i}$. Such a state can be identified by the $n$-element vector $\vec{t}=\left(t_{1}, t_{2}, \ldots, t_{n}\right)$, with $1 \leqslant t_{i} \leqslant m$. Since different orderings of the particles in the same modes are not distinguishable, we will consider only the cases $t_{1} \leqslant t_{2} \leqslant$ $\ldots \leqslant t_{n}$.

An $m$-mode lossless linear evolution can be described by an $m \times m$ unitary transformation $U$ on the space of creation operators. The probability amplitude associated with an input $\vec{g}=\left(g_{1}, g_{2}, \ldots, g_{n}\right)$ and output $\vec{h}=\left(h_{1}, h_{2}, \ldots, h_{n}\right)$ is given by

$$
p_{\text {bos }}=\frac{\operatorname{perm} S_{\vec{g}, \vec{h}}}{\sqrt{\mu_{1} ! \mu_{2} ! \ldots \mu_{m} ! \nu_{1} ! \nu_{2} ! \ldots v_{m} !}}
$$

in the case of bosons and by

$$
p_{\text {fer }}=\frac{\operatorname{det} S_{\vec{g}, \vec{h}}}{\sqrt{\mu_{1} ! \mu_{2} ! \ldots \mu_{m} ! v_{1} ! v_{2} ! \ldots v_{m} !}},
$$

where $\mu_{i}$ and $v_{i}$ are the number of particles present in mode $i$ in the $\vec{g}$ and $\vec{h}$ should have the arrow sign as they are vectors states, respectively; $S$ is the scattering matrix with elements $S_{i, j}=U_{h_{i}, g_{j}}$; and perm $A$ denotes the permanent of a matrix $A$. It may be useful to recall also the definition of permanent for a matrix $A$ :

$$
\operatorname{perm} A=\sum_{\sigma} \prod_{i=1}^{n} a_{i, \sigma(i)},
$$

where $a_{i, j}$ is an element of $A, \sigma$ is a permutation of $\{1, \ldots, n\}$, and thus the sum in the expression is performed over all the possible permutations.

\section{B. Hadamard, Fourier, and Sylvester matrices}

A complex Hadamard matrix is defined as an orthogonal matrix of complex numbers, in which all the elements have a unitary modulus. A well-known subclass of such matrices is that of Fourier matrices, the elements of an $m \times m$ Fourier matrix $F(m)$ being defined as follows:

$$
F_{j, k}=e^{2 \pi \iota(j-1)(k-1) / m},
$$

where $\iota=\sqrt{-1}$ is the imaginary unit. As already mentioned, multiparticle interference has been largely studied in the literature $[7,20,21]$ for interferometers implementing the normalized (unitary) version of such matrices $U_{m}=\frac{1}{\sqrt{m}} F(m)$.

Real Hadamard matrices, simply referred to as Hadamard matrices in the following, are orthogonal matrices with all elements equal to \pm 1 . Sylvester matrices are a particular class of real Hadamard matrices, having size $m=2^{p}$, that can be built recursively from the following formula:

$$
H\left(2^{p}\right)=\left[\begin{array}{cc}
H\left(2^{p-1}\right) & H\left(2^{p-1}\right) \\
H\left(2^{p-1}\right) & -H\left(2^{p-1}\right)
\end{array}\right],
$$

with $H\left(2^{0}\right)=H(1)=[1]$. From this construction one can derive an analytic expression for the $(i, j)$ element of the matrix:

$$
\left[H\left(2^{p}\right)\right]_{i, j}=(-1)^{i_{B} \odot j_{B}},
$$

where $i_{B}$ and $j_{B}$ are the binary representations of $i$ and $j$, enumerating the rows and columns starting from zero, and $\odot$ is the bitwise dot product.

In the following we will refer to devices implementing a unitary matrix defined by

$$
U_{m}=\frac{1}{\sqrt{m}} H(m),
$$

with $m=2^{p}$ as Sylvester interferometers.

A general expression for the permanent of Sylvester (and more generally Hadamard) matrices is not known: while perm $H(2)=0$, it has been conjectured that for all the other orders Hadamard matrices have nonvanishing permanents [22].

Note that the usual balanced beam-splitter operator is simply $\frac{1}{\sqrt{2}} H(2)$. In this case, for an input state with one photon per mode, the well-known Hong-Ou-Mandel effect is observed, which consists in the suppression of the output state with one photon per mode. In fact, according to Eq. (1), for such an output contribution, the probability amplitude is proportional to perm $H(2)=0$.

\section{TWO PARTICLES}

Even though some of the results of this section could be retrieved by applying the more general results of Secs. IV and $\mathrm{V}$, the two-particle case allows for a more comprehensive description and shows some specific features, which make it worth addressing it separately.

Proposition 1. If two bosons are injected in the first two modes of an interferometer described by $U_{m}=\frac{1}{\sqrt{m}} H(m)=$ $\frac{1}{\sqrt{2^{p}}} H\left(2^{p}\right)$, the probability amplitude $p_{i, j}$ of an output state with one particle on mode $i$ and one particle on mode $j$ follows 
the rule

$$
\begin{array}{rlrl}
\left|p_{i, j}\right| & =\frac{1}{2^{p-1}} \quad \text { if } \quad i \quad \bmod 2=j & \bmod 2, i \neq j, \\
\left|p_{i, j}\right| & =\frac{1}{2^{p-1 / 2}} & \text { if } \quad i=j, & \text { else. }
\end{array}
$$

The scattering matrices $S$ in Eq. (1), for such an input state, are all submatrices of the first two columns of $U_{m}$. Recalling Eq. (5), one can easily observe that such columns are simply (properly normalized) repetitions of $H(2)$ and retrieve the matrices $S$, as a function of $i$ and $j$, as follows:

(1) $i \bmod 2=1$ and $j \bmod 2=0$, $S=\frac{1}{\sqrt{2^{p}}} H(2)=\frac{1}{\sqrt{2^{p}}}\left[\begin{array}{cc}1 & 1 \\ 1 & -1\end{array}\right] \Rightarrow \operatorname{perm} S=0$.

(2) $i \bmod 2=0$ and $j \bmod 2=1$, $S=\frac{1}{\sqrt{2^{p}}}\left[\begin{array}{cc}1 & -1 \\ 1 & 1\end{array}\right] \Rightarrow \operatorname{perm} S=0$.

(3) $i \bmod 2=j \bmod 2$.

$S$ consists of two identical rows with elements $\pm \frac{1}{\sqrt{2^{p}}}$ : simple calculations show that in this case $\mid$ perm $S \mid=\frac{1}{2^{p-1}}$.

Thus, if and only if $i \bmod 2 \neq j \bmod 2$ the permanent of the scattering matrix vanishes, giving $p_{i, j}=0$, while the other cases are proved by applying Eq. (1).

Corollary 1.1. In the case of Proposition 1, the fraction of suppressed states is $\frac{N_{\text {supp }}^{\text {bos }}}{N_{\text {states }}}=\frac{1}{2} \frac{m}{m+1}$.

The possible two-bosons output states are identified by all the couples $(i, j)$ with $1 \leqslant i \leqslant j \leqslant m$ [we consider only $i \leqslant j$ because $(i, j)$ is the same state as $(j, i)]$. The number of such states is $\frac{1}{2} m(m+1)$. In a chessboard with $m \times m$ squares, alternately black and white, these can be seen as all the squares above the main diagonal or included in it. If we color the squares in such a way that the main diagonal is black, the condition $i \bmod 2 \neq j \bmod 2($ with $i \leqslant j$ ) indicates all the white squares comprised in the region above it, which are actually half of the total number of white squares. Thus, the number of suppressed states is $\frac{1}{4} m^{2}$, giving the result.

Proposition 2. If two fermions are injected in the first two modes of an interferometer described by $U_{m}=\frac{1}{\sqrt{m}} H(m)=$ $\frac{1}{\sqrt{2^{p}}} H\left(2^{p}\right)$, the probability amplitude $p_{i, j}$ of an output contribution with one particle on mode $i$ and one particle on mode $j$ follows the rule

$$
\begin{array}{rlrl}
\left|p_{i, j}\right| & =\frac{1}{2^{p-1}} \quad \text { if } \quad i \quad \bmod 2 \neq j \quad \bmod 2, \\
p_{i, j} & =0 & & \text { else. }
\end{array}
$$

The scattering matrices to be considered for calculating the probability amplitudes are the same as those of Proposition 1, but the determinant [Eq. (2)] instead of the permanent has to be calculated here. Thus, when $i \bmod 2 \neq j \bmod 2$ the scattering matrix is composed of two identical rows and the determinant vanishes. In all the other cases (see the expression of $S$ in the proof of Proposition 1) the determinant is equal to $\frac{1}{2^{p-1}}$. Application of Eq. (2) then gives the probability amplitudes.

Corollary 2.1. In the case of Proposition 2 the fraction of suppressed states is $\frac{N_{\text {sup }}^{\text {fer }}}{N_{\text {states }}}=\frac{1}{2} \frac{m+2}{m+1}$.
Comparing Proposition 1 with Proposition 2, bosons and fermions show a dichotomic behavior, in that an output combination is suppressed for two bosons if and only if it is allowed for two fermions and vice versa. Thus the fraction of suppressed states (over all the possible two-particle states) for two fermions is $\frac{N_{\text {susp }}^{\text {fer }}}{N_{\text {states }}}=1-\frac{N_{\text {susp }}^{\text {bos }}}{N_{\text {states }}}=1-\frac{1}{2} \frac{m}{m+1}=\frac{1}{2} \frac{m+2}{m+1}$.

Note that this fraction actually includes some states (the states with two particles on the same port) that are indeed suppressed by virtue of the Pauli principle and not by specific features of the Sylvester matrix.

Proposition 3. For $U_{2^{p}}=\frac{1}{\sqrt{2^{p}}} H\left(2^{p}\right)$ and a two-particle input on an arbitrary couple of different modes, the number of suppressed states is the same as that given in Corollaries 1.1 and 2.1 for bosons and fermions, respectively.

For an arbitrary input state, with two particles on modes $(i, j)$, the scattering matrices $S$ will take elements from the $i$ th and $j$ th columns of $\frac{1}{\sqrt{2^{p}}} H\left(2^{p}\right)$. Let us put these two columns one next to the other, to form the $n \times 2$ matrix $A$. For a given output state $\left(i^{\prime}, j^{\prime}\right)$, the scattering matrix $S$ will be a submatrix of $A$ formed by its $i^{\prime}$ th and $j^{\prime}$ th rows.

Note that two different columns of a Hadamard matrix have half of the elements with opposite sign and half of the elements with the same sign. Half of the rows of $A$ will be $[1,1]$ or $[-1,-1]$; the other half will be $[-1,1]$ or $[1,-1]$. Let us now perform the following operations. First, we multiply the $[-1,-1]$ and $[-1,1]$ rows by -1 . This will change the sign of the permanent of the scattering matrix that should include such rows, but it has no influence if the permanent vanishes. At this point we will have half of the rows equal to $[1,1]$ and half equal to $[1,-1]$. Second, we reorder the rows alternating $[1,1]$ and $[1,-1]$. This is equivalent to relabeling the outputs, which does not affect the number of suppressed output states. At this point the matrix $A$ will be the same as if $(i, j)=(1,2)$, which is the case of Propositions 1 and 2. Hence, every input combination has the same fraction of suppressed output states of the input $(i, j)=(1,2)$, discussed in Corollaries 1.1 and 2.1 for boson and fermions, respectively.

\section{MULTIPLE BOSONS}

The aim of this section is to demonstrate a suppression law for the case of $n=2^{q}$ indistinguishable bosons. This will be obtained in Proposition 7 at the end of the section. However, that result is based on other propositions which will be proved first. The first one (Proposition 4) allows us to restrict the study, in certain conditions, from the case of $n$ particles in $m$ modes to the case of $n$ particles in $n$ modes. Propositions 5 and 6 , in contrast, regard mathematical properties (in particular, the value of the permanent) of certain $-1,+1$ matrices.

Proposition 4. Let $U=\frac{1}{\sqrt{2^{p}}} H\left(2^{p}\right)$ with $p=k+q$ be a linear transformation over $m=2^{p}$ modes, and let $\vec{h}=(1+$ $n c, \ldots, n+n c)$ with $0 \leqslant c \leqslant\left(2^{k}-1\right)$ be an input state of $n=$ $2^{q}$ particles. The output state $\vec{g}=\left(g_{1}, \ldots, g_{n}\right)$ is suppressed if and only if the output state $\vec{g}^{\prime}=\left(g_{1}^{\prime}, \ldots, g_{n}^{\prime}\right)$ with $g_{i}^{\prime}=$ $\left[\left(g_{i}-1\right) \bmod n\right]+1$ is suppressed for the transformation $U^{\prime}=\frac{1}{\sqrt{2^{q}}} H\left(2^{q}\right)$ with $n$ particles entering one per each mode.

For input states of the kind $\vec{h}=(1, \ldots, n)$ (i.e., one particle per each of the first $n$ modes), the scattering matrices will be 
submatrices of the first $n$ columns of $U$. From the construction of $H\left(2^{p}\right)=H\left(2^{k+q}\right)=H\left(n \times 2^{k}\right)$ with Eq. (5), it is clear that these first $n$ columns are simply repetitions of $H(n)$. For an output state with $n$ particles distributed on the modes $g_{1} \ldots g_{n}$, the $i$ th row of the scattering matrix $S$ will be extracted from the $g_{i}$ th row of $U$. Since such rows repeat identically every $n$ rows (regarding the first $n$ columns), the scattering matrix is the same for all states $\vec{g}=\left(g_{1}, \ldots, g_{n}\right)$ having the same $g_{1} \bmod n, \ldots, g_{n} \bmod n$. We can look for the smallest mode numbers giving this condition, which are $g_{i}^{\prime}=\left[\left(g_{i}-1\right)\right.$ $\bmod n]+1$. In that case the scattering matrix is the one we would expect for the output state $\vec{g}^{\prime}=\left(g_{1}^{\prime}, \ldots, g_{n}^{\prime}\right)$ defined as above, when entering with $n$ particles (one per each mode) in an interferometer implementing $U^{\prime}=\frac{1}{\sqrt{2^{q}}} H\left(2^{q}\right)$.

Let us consider now the more general case $\vec{h}=(1+$ $n c, \ldots, n+n c)$ with $0 \leqslant c \leqslant 2^{k-1}$. Again from the construction in Eq. (5) it can be observed that such columns will be repetitions of $\pm H(n)$ (with a succession of positive and negative signs that depends on $c$ ). If we properly change the signs of the rows (an operation that is equivalent to adding a $\pi$ phase term to certain outputs, which does not influence the probability modulus) these columns can be made identical to those of the case $\vec{h}=(1, \ldots, n)$, discussed above. Hence, the output distribution is the same.

This result holds for both bosons and fermions because no hypotheses on the particle statistics have been adopted. In addition it can be exploited for a more precise generalization of the results of Propositions 1 and 2 to a wider range of input states.

Proposition 5. Let $A$ be an $m \times m$ matrix with $m=2^{p}$, built by taking the rows $\left\{r_{1}, r_{2}, \ldots, r_{m}\right\}$ from $H(m)$ [namely, the $i$ th row of $A$ is the $r_{i}$ th row of $H(m)$ and rows may be repeated]. If $r_{1, B} \oplus r_{2, B} \oplus \ldots \oplus r_{m, B} \neq 0$, then perm $A=0, r_{i, B}$ being the binary representation of the row number, starting the count from zero, and $\oplus$ being the bitwise sum (the XOR operation).

The condition $r_{1, B} \oplus r_{2, B} \oplus \ldots \oplus r_{m, B} \neq 0$ means that for at least one $k$ an odd number of $r_{i, B}$ has the same $k$ th bit. In other words, for at least one $k$, the $k$ th bit of the binary representations of the $r_{i}$ is 1 for an odd number of rows and is zero for a (possibly different) odd number of rows.

Consider now an arbitrary permutation $\sigma$ in the permanent expression perm $A=\sum_{\sigma} \prod_{i=1}^{n} a_{i, \sigma(i)}$, which is actually a set $\{\sigma(i)\}$ containing the numbers from 1 to $n$ in a certain order; further, let $\sigma^{\prime}$ be another permutation, obtained from $\sigma$ by changing the $k$ th bit in all its components $\sigma(i)$ (written in their binary representation). Let us analyze the effect of this bit flip. First, one should recall that, from its definition,

$$
a_{i, j}=(H(m))_{r_{i}, j},
$$

with $\left|a_{i, j}\right|=1 \quad \forall i, j$. Depending on the value of the $k$ th bit of $r_{i, B}$, one has from Eq. (6)

$$
\begin{aligned}
\left.r_{i, B}\right|_{k} & =1 \Rightarrow a_{i, \sigma(i)}=(H(m))_{r_{i}, \sigma(i)} \\
& =-(H(m))_{r_{i}, \sigma^{\prime}(i)}=-a_{i, \sigma^{\prime}(i)}, \\
\left.r_{i, B}\right|_{k} & =0 \Rightarrow a_{i, \sigma(i)}=(H(m))_{r_{i}, \sigma(i)} \\
& =(H(m))_{r_{i}, \sigma^{\prime}(i)}=a_{i, \sigma^{\prime}(i)} .
\end{aligned}
$$

If, as in the case of the hypotheses, an odd number of $r_{i, B}$ have the $k$ th bit equal to 1 , in the product $\prod_{i=1}^{n} a_{i, \sigma^{\prime}(i)}$ an odd number of factors change their sign with respect to $\prod_{i=1}^{n} a_{i, \sigma(i)}$, giving

$$
\prod_{i=1}^{n} a_{i, \sigma(i)}=-\prod_{i=1}^{n} a_{i, \sigma^{\prime}(i)} .
$$

This means that for each permutation $\sigma$ there exists another $\sigma^{\prime}$, biunivocally associated to $\sigma$, for which Eq. (11) holds. Hence, in the sum over all the $\sigma$ of Eq. (3), half of the addends will have sign -1 and the other half will have sign +1 , which implies perm $A=0$.

An example of an application of this criterion is given in Fig. 1.

Corollary 5.1. Let $A$ be an $m \times m$ matrix with $m=2^{p}>2$, built by taking the rows $\left\{r_{1}, r_{2}, \ldots, r_{m}\right\}$ from $H(m)$ [namely, the $i$ th row of $A$ is the $r_{i}$ th row of $H(m)$ and rows may be repeated], and $r_{1, B} \oplus r_{2, B} \oplus \ldots \oplus r_{m, B}=0$. Build the matrix $B$ such that

\begin{tabular}{c|c|c|c}
$i$ & $r_{i}$ & $r_{i, B}$ & \\
\hline 1 & 1 & 000 & \\
2 & 2 & 001 & \\
4 & 3 & 010 & \\
5 & 3 & 010 & \\
6 & 5 & 100 & \\
7 & 6 & 101 \\
8 & 7 & 110 & \\
& 8 & 101 & \\
\hline$r_{1, B} \oplus r_{2, B} \oplus \ldots \oplus r_{n, B}$ & & 011 & $\neq 000$
\end{tabular}

FIG. 1. Example of application of the criterion of Proposition 5, for a matrix $A$ built of the rows $\{1,2,3,3,5,6,7,8\}$ of $H(8)$. The table on the right summarizes the application of the criterion: for each row $i$ of the scattering matrix, which is the $r_{i}$ line of $H(8)$, the binary expression $r_{i, B}$ is reported. The latter is actually the binary conversion of $r_{i}-1$, since the binary enumeration of the rows must start from zero. The last line of the table reports the bitwise sum which, being not equal to zero in this case, indicates that perm $A=0$. 
all the rows are the same as those of $A$ except the $i$ th row, the $i$ th row being another arbitrary $r_{i}^{\prime}$ th row of $H(m)$, with $r_{i}^{\prime} \neq r_{i}$. Then, $B$ satisfies Proposition 5.

The condition $r_{1, B} \oplus r_{2, B} \oplus \ldots \oplus r_{m, B}=0$ means that in the set of the binary representations $\left\{r_{1, B}, r_{2, B}, \ldots, r_{m, B}\right\}$ each bit recurs an even number of times with value zero and an even number of times with value one. The matrix $B$ is built by removing from $A$ its $i$ th row [which was the $r_{i}$ th row of $H(m)$ ] and by replacing it with another arbitrary $r_{i}^{\prime}$ th row of $H(m)$. The binary representation $r_{i, B}^{\prime}$ is different from $r_{i, B}$ for at least one bit, say, the $k$ th bit. Note that in the set $\left\{r_{1, B}, r_{2, B}, \ldots, r_{m, B}\right\}$ the $k$ th bit had the value zero for an even number of times and the value one for an even number of times: now that we have changed $r_{i}$ with $r_{i}^{\prime}$ the $k$ th bit will have the value zero for an odd number of times and the value one for (another) odd number of times. This implies $r_{1, B} \oplus r_{2, B} \oplus \ldots \oplus r_{i, B}^{\prime} \oplus \ldots \oplus r_{n, B} \neq 0$ and the hypotheses for Proposition 5 are verified.

Proposition 6. Let $A$ be an $m \times m$ matrix with $m=2^{p}>2$, built by taking the rows $\left\{r_{1}, r_{2}, \ldots, r_{n}\right\}$ from $H(m)$ (namely, the $i$ th row of $A$ is the $r_{i}$ th row of $H(m)$ and rows may be repeated). If $r_{1, B} \oplus r_{2, B} \oplus \ldots \oplus r_{n, B}=0$, then perm $A \neq 0$.

Take the Laplace expansion of the permanent along an arbitrary $i$ th row:

$$
\operatorname{perm} A=\sum_{j} a_{i, j} \times \operatorname{perm} M_{i, j},
$$

where $a_{i, j}$ is an element of $A$ and $M_{i, j}$ is the $i, j$ minor of $A$. In other terms, Eq. (12) can be read as a dot product:

$$
\operatorname{perm} A=\vec{a}_{i} \cdot \vec{c},
$$

where $\vec{a}_{i}$ is the $n$-element vector given by the $i$ th row of $A$ and $\vec{c}$ is the vector with elements $c_{j}=$ perm $M_{i, j}$.

Consider now $m$ different matrices $A_{l}$, built by replacing the $i$ th row of $A$ with the $l$ th row of $H(m)$. For $l=r_{i}$ one has $A_{l}=A_{r_{i}}=A$, while all the other $A_{l}$ will differ from $A$ by one row. When one calculates perm $A_{l}$ according to Eq. (12), the minors $M_{i, j}$ are always the same for every $A_{l}$, because only the $i$ th row is changing. Hence, in Eq. (13) the vector $\vec{c}$ is always the same for every $A_{l}$. The permanents of the different $A_{l}$ can be interpreted as the projection of such $\vec{c}$ onto different vectors, given by the $l$ th row of $H(m)$.

It is important to note that $\vec{c}$ is a nonzero vector. The elements of this vector are permanents of matrices $M_{i, j}$, which are squared $(+1,-1)$ matrices of order $m-1$, and it has been shown [23] that if $m=2^{p}$ then no matrices of order $m-1$ exist with a vanishing permanent.

The rows of $H(m)$ form a complete (orthogonal) basis of $\mathbb{R}^{n}$ : a nonzero vector $\vec{c}$ has at least one nonzero projection on one of the vectors of the basis. We have already shown (Proposition 5.1) that, for $A_{l}$ defined as above with $l \neq r_{i}$, one has perm $A_{l}=0$; i.e., the projection of $\vec{c}$ on all the rows of $H(m)$, except the $r_{i}$ th, is vanishing. It follows that the projection on the $r_{i}$ th row must be nonzero: this implies perm $A_{r_{i}}=\operatorname{perm} A \neq 0$.

Proposition 7. Consider a unitary transformation $m=2^{p}$ modes $U_{m}=\frac{1}{\sqrt{2^{p}}} H\left(2^{p}\right)$ with $p=k+q$ and an input state with $n=2^{q}$ bosons $\vec{h}=(1+n c, \ldots, n+n c)$, where $0 \leqslant c \leqslant$ $\left(2^{k}-1\right)$. The output state $\vec{g}=\left(g_{1}, g_{2}, \ldots, g_{n}\right)$ is suppressed if and only if $g_{1, B} \oplus g_{2, B} \oplus \ldots \oplus g_{n, B} \neq 0, g_{i, B}$ being the binary representation of $g_{i}-1$ (i.e., the binary representation of the output mode number, starting the count from zero) truncated to the $q$ least significant bits and $\oplus$ being the bitwise sum (the XOR operation).

First we address the case $m=n$, with $\vec{h}=(1, \ldots, n)$. Here the result comes directly from considering that the probability of an output configuration $\vec{g}=\left(g_{1}, g_{2}, \ldots, g_{n}\right)$ is proportional to perm $S$, where $S$ is a matrix whose $i$ th row is the $g_{i}$ th row of $H(n)$. Because of Propositions 5 and 6 such a permanent is vanishing if and only if $g_{1, B} \oplus g_{2, B} \oplus \ldots \oplus g_{n, B} \neq 0$ (where $g_{i, B}$ is the full binary expression of $g_{i}-1$, composed of $q$ bits), thus giving in this case the suppression of the corresponding output configuration.

By exploiting Proposition 4, this result can now be extended to the case $m=2^{q}>2^{p}=n$ and input states of the kind $\vec{h}=(1+n c, \ldots, n+n c)$. In particular, the condition of Proposition 4 of considering the mode numbers modulo $n$ implies that a criterion of the kind $g_{1, B} \oplus g_{2, B} \oplus \ldots \oplus g_{n, B} \neq$ 0 can be applied if $g_{i, B}$ is the binary representation of the mode index, truncated to the $q$ least significant bits.

To evaluate the fraction of output combinations that is suppressed we need to consider the set of all possible output states $\left\{g_{1}, g_{2}, \ldots, g_{n}\right\}$ and estimate when their binary expressions $\left\{g_{1, B}, g_{2, B}, \ldots, g_{n, B}\right\}$, truncated to the $q$ least significant bits (for the arguments discussed above), satisfy $G=g_{1, B} \oplus$ $g_{2, B} \oplus \ldots \oplus g_{n, B} \neq 0$. Adopting an approach similar to that of Ref. [7], we assume that in such a set a certain $k$ th bit of the binary expression $g_{i, B}$ (consisting of $q$ bits) can take the values zero or one with equal probability, independently from the values of the other bits. In other words, we assume that in each subset of states with a certain bit combination (for the bits other than the $k$ th bit) the number of output states for which the $k$ th bit is zero is equal to the number of states for which that bit is one.

Let us now consider the possible values of the binary expression $G$, starting from its first bit. That bit is the result of the $\oplus$ operation on $n$ bits (the first bit of each $g_{i, B}$ ). If we consider the full set of possible outputs, such $n$ bits will be zero or one the same number of times. Thus, also the first bit of $G$ will be zero for half of the possible output states and one for the other half. Those states for which the first bit of $G$ is 1 already satisfy $G \neq 0$, so they are suppressed. For the other ones, they may be suppressed if the $\oplus$ operation on other bits gives 1 . One then considers the second bit and with analogous arguments notes that it will be zero for the half of the output states and one for the other half. One continues with the same procedure up to the $q$ th bit. Hence, the overall fraction $\frac{N_{\text {supp }}^{\text {bos }}}{N_{\text {states }}}$ of suppressed states will be $\frac{1}{2}$ (the fraction of states which has the first bit of $R$ equal to 1 ) summed to $\frac{1}{2} \times \frac{1}{2}$ (the fraction of states which has the first bit of $R$ equal to zero and the second equal to one) summed to $\frac{1}{2} \times \frac{1}{2} \times \frac{1}{2}$ (the fraction of states which has the first and second bit equal to zero and the third equal to one) and so on. This gives

$$
\frac{N_{\text {supp }}^{\text {bos }}}{N_{\text {states }}} \sim \sum_{x=1}^{k} \frac{1}{2^{x}}=\frac{2^{k}-1}{2^{k}}=\frac{n-1}{n} .
$$

Table I reports the fraction of suppressed bosonic states for $n \leqslant 8$ and $m \leqslant 64$, compared with the result of Eq. (14). 
TABLE I. Fraction of suppressed states over the possible output states, when injecting an $m$-modes Sylvester interferometer with $n$ bosons in the first $n$ inputs. The number of suppressed states has been calculated by checking the criterion of Proposition 4 for each possible output state. In the last column the estimation from the formula (14) is given for comparison.

\begin{tabular}{|c|c|c|c|c|c|c|c|}
\hline \multirow[b]{2}{*}{$n$} & \multicolumn{6}{|c|}{$m$} & \multirow[b]{2}{*}{$\frac{n-1}{n}$} \\
\hline & 2 & 4 & 8 & 16 & 32 & 64 & \\
\hline 2 & $\frac{1}{3} \simeq 0.33$ & $\frac{4}{10}=0.4$ & $\frac{16}{36} \simeq 0.44$ & $\frac{64}{136} \simeq 0.47$ & $\frac{256}{528} \simeq 0.48$ & $\frac{1,024}{2,080} \simeq 0.49$ & 0.5 \\
\hline 4 & & $\frac{24}{35} \simeq 0.69$ & $\frac{240}{330} \simeq 0.73$ & $\frac{2,880}{3,876} \simeq 0.74$ & $\frac{39,168}{52,360} \simeq 0.75$ & $\frac{574,464}{766,480} \simeq 0.75$ & 0.75 \\
\hline 8 & & & $\frac{5,600}{6,435} \simeq 0.870$ & $\frac{428,736}{490,314} \simeq 0.874$ & $\frac{53,829,888}{61,523,748} \simeq 0.875$ & $\frac{9,309,189,120}{10,639,125,640} \simeq 0.875$ & 0.875 \\
\hline
\end{tabular}

The latter expression approximates better the actual value for increasing $n$ or $m$.

\section{MULTIPLE FERMIONS}

The case of fermions is less significant, with respect to bosons, from a computational point of view; in fact the probability amplitudes of the output configurations are proportional to the determinant of the scattering matrix [see Eq. (2)], which differently from the permanent can be calculated efficiently. However, investigating the suppression laws arising for this kind of particles enables a better understanding of the effects of statistics in multiparticle interference.

Proposition 8. Consider a unitary transformation over $m=$ $2^{p}$ modes $U_{m}=\frac{1}{\sqrt{2^{p}}} H\left(2^{p}\right)$ with $p=k+q$ and an input state with $n=2^{q}$ fermions $\vec{h}=(1+n c, \ldots, n+n c)$, where $0 \leqslant$ $c \leqslant\left(2^{k}-1\right)$. The output state $\vec{g}=\left(g_{1}, \ldots, g_{n}\right)$ is suppressed if and only if $g_{i} \bmod n=i \bmod n \quad \forall i \in[1, n]$.

Let us consider, to begin, the case $n=m$, i.e., $n$ fermions entering an $n$-mode interferometer one per each port. The only possible output state allowed from the Pauli principle is $\vec{g}=(1, \ldots, n)$, namely, the state having one particle per mode, which can be written also as $g_{i}=i \quad \forall i \in[1, n]$. Such a condition is easily extended to the case of a more generic input state $\vec{h}=(1+n c, \ldots, n+n c)$ of $n=2^{q}$ fermions entering an $m=n \times 2^{k}$ interferometer through Proposition 4, becoming $g_{i} \bmod n=i \bmod n \quad \forall i \in[1, n]$.

The number of allowed and suppressed output states can be evaluated considering that, in an interferometer with $m=$ $n \times 2^{k}$ modes, the condition $g_{i} \bmod n=i \bmod n$ can be satisfied for $2^{k}$ different values of $g_{i}$. Hence, the number of allowed output states is the number of sequences of $n$ numbers, each with $2^{k}$ possible values, i.e., $2^{k n}=\left(\frac{m}{n}\right)^{n}$.

The possible output states of $n$ particles on $m$ modes are $N_{\text {states }}=\left(\begin{array}{c}m+n-1 \\ n\end{array}\right)=\frac{(m+n-1) !}{(m-1) ! n !}$ (combinations with repeti- tions). The fraction of allowed states, for large $m$, thus tends to [24]

$$
\left(\frac{m}{n}\right)^{n} \frac{(m-1) ! n !}{(m+n-1) !} \asymp\left(\frac{m}{m-1}\right)^{n} \frac{n !}{n^{n}} \asymp \frac{n !}{n^{n}},
$$

and the fraction of suppressed states is asymptotically equal to

$$
\frac{N_{\text {supp }}^{\text {fer }}}{N_{\text {states }}}=1-\frac{N_{\text {allowed }}^{\text {fer }}}{N_{\text {states }}} \asymp 1-\frac{n !}{n^{n}} .
$$

Table II reports the fraction of suppressed fermionic states for $n \leqslant 8$ and $m \leqslant 64$, calculated over all the possible $n$ particles states, together with the asymptotic estimation with Eq. (16).

\section{DISCUSSION}

The predictions of the suppressions laws proved above can be compared with the general trends that usually distinguish the particles' behavior, depending on their statistics. While for fermions the compatibility of Proposition 8 with the Pauli principle is intrinsic in its same proof, the behavior of bosons, which would be expected to show an enhanced tendency to bunch together, is more peculiar. Actually, similarly to Ref. [7], we observe that many bunching events are not enhanced but included in the suppression conditions of Proposition 7. The probability of full-bunching events (events with all $n$ bosonic particles on the same output mode, over the $m$ possible modes) can be calculated easily: the scattering matrix is composed of identical rows with half $+1 / \sqrt{m}$ and half $-1 / \sqrt{m}$ elements; by inverting the sign of the columns with negative elements (an operation which does not affect the permanent [25]), we obtain a matrix of all $+1 / \sqrt{m}$, whose permanent equals $n ! / m^{m / 2}$. Thus the probability of this event is $\left(\frac{n !}{m^{m / 2} \sqrt{n !}}\right)^{2}=n ! / m^{m}$ [from squaring Eq. (1)], which is an enhancement of $n$ ! with respect to the probability of such an event for distinguishable particles. This enhancement factor

TABLE II. Fraction of suppressed states over the possible output states, when injecting an $m$-modes Sylvester interferometer with $n$

\begin{tabular}{|c|c|c|c|c|c|c|c|}
\hline \multirow[b]{2}{*}{$n$} & \multicolumn{6}{|c|}{$m$} & \multirow[b]{2}{*}{$1-\frac{n !}{n^{n}}$} \\
\hline & 2 & 4 & 8 & 16 & 32 & 64 & \\
\hline 2 & $\frac{2}{3} \simeq 0.67$ & $\frac{6}{10}=0.6$ & $\frac{20}{36} \simeq 0.56$ & $\frac{72}{136} \simeq 0.53$ & $\frac{272}{528} \simeq 0.52$ & $\frac{1,056}{2,080} \simeq 0.51$ & 0.5 \\
\hline 4 & & $\frac{34}{35} \simeq 0.97$ & $\frac{314}{330} \simeq 0.95$ & $\frac{3,620}{3,876} \simeq 0.93$ & $\frac{48,264}{52,360} \simeq 0.92$ & $\frac{700,944}{766,480} \simeq 0.91$ & 0.91 \\
\hline 8 & & & $\frac{6,434}{6,435}>0.999$ & $\frac{490,058}{490,314}>0.999$ & $\frac{61,458,212}{61,523,748} \simeq 0.999$ & $\frac{10,622,348,424}{10,639,125,640} \simeq 0.998$ & 0.998 \\
\hline
\end{tabular}
fermions in the first $n$ inputs. In the last column the asymptotic value $1-\frac{n !}{n^{n}}$ is given for comparison. 

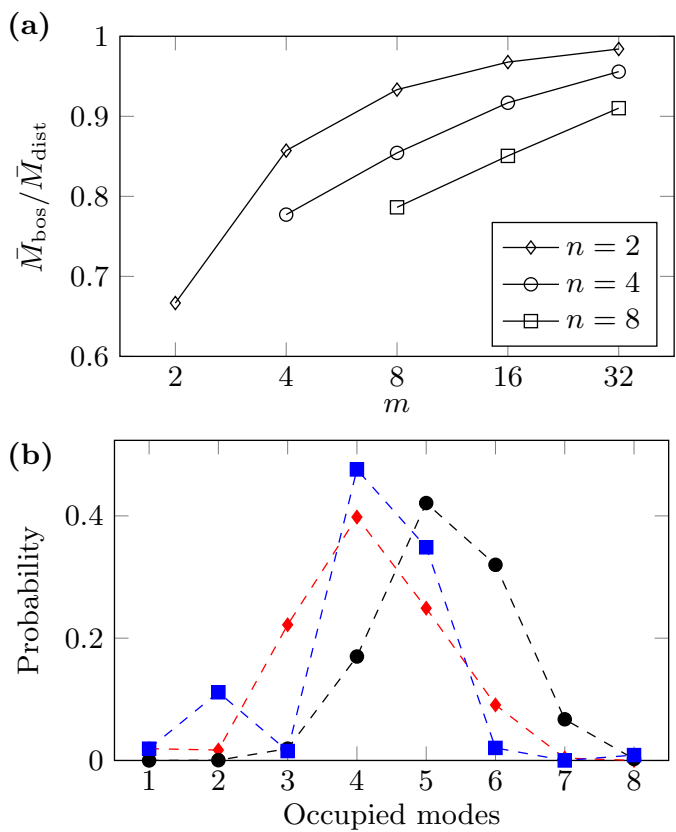

FIG. 2. (Color online) (a) Ratio between the average number of occupied modes for bosons and for distinguishable particles, for $n=2,4,8$ particles entering an $m$-mode Sylvester interferometer, one per each of the first $n$ modes. (b) Probability distribution of detecting an output state with a certain number of occupied modes, when entering with $n=8$ indistinguishable bosons (one per each input mode) in a $m=8$ Sylvester interferometer (blue squares) or Fourier interferometer (red diamonds). Probability for distinguishable particles (black circles) in analogous interferometers is also reported.

for full-bunching events is indeed a general law for all unitary processes [3]. With regard to antibunching events (particles in all different ports), it is not difficult to observe that for $n=m>2$ they are instead never suppressed for bosons: the scattering matrix for such an event would be a full Sylvester matrix, whose permanent is proved [26] to be nonvanishing by observing that for $\left\{r_{1}, r_{2}, \ldots, r_{n}\right\}=\{1,2, \ldots, n\}$ one has $r_{1, B} \oplus r_{2, B} \oplus \ldots \oplus r_{n, B}=0$ and by exploiting Proposition 6 . This also marks a difference with respect to Bell multiports, where such events are always suppressed for an even number of bosons [20].

An overall figure that may quantify the bunching behavior [7] is the average number $\bar{M}$ of occupied modes at the output. The bosonic bunching tendency should reduce the number of occupied modes with respect to the case of distinguishable particles. Figure 2(a) reports the ratio between the average number of occupied modes in the case of indistinguishable bosons and that in the case of distinguishable particles, for $n=2,4$, and 8 particles injected in the first modes of Sylvester interferometers with up to 32 modes. This ratio is always smaller than unity, confirming the bunching behavior. However, while from the previous discussion we know that the fraction of suppressed states is practically constant with increasing $m$, this ratio looks to approach 1 in the same limit: the larger number of available modes makes the particles more likely to exit on different ports, approaching the classical probability on this aspect. Thus, the suppression law seems to be a stronger nonclassical signature than the bunching behavior itself, which is less evident with large $m$.

The detailed probability of having a certain number of occupied modes is reported in Fig. 2(b) for the case $n=m=$ 8 , with the comparison of the distribution in the case of a Fourier interferometer. The three distributions are different in shape and the shift toward a smaller number of occupied modes for the two nonclassical distributions is evident. In this particular case the average number of occupied ports is $\simeq 4.1$ for both Sylvester and Fourier interferometers in the case of identical bosons and is $\simeq 5.3$ in the case of distinguishable particles.

As a further analysis, the asymptotic fraction of allowed fermionic states can be compared with the asymptotic fraction of allowed bosonic states, to evaluate the strength of the suppression law in the two cases. For large $m$, one has

$$
\frac{N_{\text {allowed }}^{\text {fer }}}{N_{\text {states }}} \asymp \frac{n !}{n^{n}}=\frac{n}{n} \frac{n-1}{n} \ldots \frac{1}{n}<\frac{1}{n} \asymp \frac{N_{\text {allowed }}^{\text {bos }}}{N_{\text {states }}} .
$$

Interestingly, the suppression law seems to act more severely for fermions, for large $m$. Note that the calculation in Eq. (17) actually includes, for fermions, all the possible multiparticle states, even those already forbidden from the Pauli principle itself. It can be observed, however, that $N_{\text {states }}^{\text {fer }}=\left(\begin{array}{c}m \\ n\end{array}\right) \asymp$ $\left(\begin{array}{c}m+n-1 \\ n\end{array}\right)=N_{\text {states }}$ for large $m$, where $N_{\text {states }}^{\text {fer }}$ are all the possible fermionic states, i.e., states with all different output ports. Thus the inequality (17) holds asymptotically also considering (for fermions) only the fraction of events that were not already suppressed by the simple application of the Pauli principle. It is worth recalling that, besides manifesting naturally for true fermions and bosons, the effects of the statistics can be simulated by proper entangled states [27,28]. Thus, the laws here developed for the two kinds of particles hold true for the corresponding entangled states.

From a more applicative point of view, suppression laws such as the one presented here for Sylvester interferometers may be exploited to test the indistinguishability of $n$-photon sources [7]. Further, they may be used, in the context of boson sampling experiments, to simultaneously check the quality of the sources and of a possible reconfigurable device [18], that would perform the required unitary that expresses the suppression law. The limitation of the laws for Sylvester interferometers to specific values of $n$ may seem, at first glance, quite disadvantageous in a possible general case when it may be required to test a general $n$-photon source where $n$ may not be a power of 2 . However, one may envisage that the full $n$-photon interference could be used as an overall check of the source quality, but an accurate troubleshooting of possible malfunctioning or imperfections requires different subsets of single-photon sources to be tested separately. To this purpose one may configure a device to implement a block-diagonal matrix, having for each block a different $2^{p}$-mode (Sylvester) unitary to test separately different subsets of single photons.

An interesting perspective to test at the same time the indistinguishability of different couples of photons, without the need of reconfiguring the device, is given by Proposition 3 , which is characteristic of Sylvester matrices and does not hold for Fourier ones. In fact, in the two-photon case, whichever couple of inputs is excited, an identical fraction of outputs 
is suppressed. This may be particularly useful to check single-photon sources for scattershot boson sampling [29]: there, several heralded single-photon sources are used, each coupled to a different input port, and multiphoton states (with one photon per port, but multiple ports excited) are generated randomly. Couples of photons on random input ports are generated efficiently in such a setup and by comparing the detected output events with the predictions of Proposition 3 the indistinguishability of all the possible couples of sources may be conveniently tested.

\section{CONCLUSION}

In conclusion, we have proved a necessary and sufficient criterion for the suppression of many output combinations when $n=2^{q}$ particles are injected in certain inputs of a linear interferometer with $m=2^{p}$ modes implementing a Sylvester matrix. While both the bosonic and fermionic cases have been studied, the result is particularly significant for bosons, whose output distribution is hard to compute in the general case. Therefore, this suppression law may be exploited for the use of Sylvester multiports as benchmark devices for the indistinguishability of multiple single-photon sources or the assessment of the overall quality of reconfigurable interferometers.

This study has also shown that comprehensive laws that describe the output multiphoton distribution of multiport interferometers on the basis of the symmetry of the implemented matrix are not limited to Fourier ones. Indeed, further investigations could pursue the definition of similar criteria for a wider class of matrices, thus giving greater insight on the features of multiparticle interference.

\section{ACKNOWLEDGMENTS}

The author acknowledges financial support from the European Research Council Starting Grant 3D-QUEST (3DQuantum Integrated Optical Simulation), Grant No. 307783.
[1] M. D. Girardeau, Phys. Rev. 139, B500 (1965).

[2] A. Arkhipov and G. Kuperberg, arXiv:1106.0849.

[3] N. Spagnolo, C. Vitelli, L. Sansoni, E. Maiorino, P. Mataloni, F. Sciarrino, D. J. Brod, E. F. Galvão, A. Crespi, R. Ramponi, and R. Osellame, Phys. Rev. Lett. 111, 130503 (2013).

[4] J. Carolan, J. Meinecke, P. Shadbolt, N. Russell, N. Ismail, K. Worhoff, T. Rudolph, M. G. Thompson, J. L. O’Brien, J. C. F. Matthews, and A. Laing, Nature Photon. 8, 621 (2014).

[5] Z. Y. Ou, J.-K. Rhee, and L. J. Wang, Phys. Rev. Lett. 83, 959 (1999).

[6] R. A. Campos, Phys. Rev. A 62, 013809 (2000).

[7] M. C. Tichy, M. Tiersch, F. de Melo, F. Mintert, and A. Buchleitner, Phys. Rev. Lett. 104, 220405 (2010).

[8] N. Spagnolo, C. Vitelli, L. Aparo, P. Mataloni, F. Sciarrino, A. Crespi, R. Ramponi, and R. Osellame, Nature Commun. 4, 1606 (2013).

[9] S. Aaronson and A. Arkhipov, in Proceedings of the FortyThird Annual ACM Symposium on Theory of Computing (ACM, New York, 2011), pp. 333-342.

[10] J. B. Spring, B. J. Metcalf, P. C. Humphreys, W. S. Kolthammer, X.-M. Jin, M. Barbieri, A. Datta, N. ThomasPeter, N. K. Langford, D. Kundys et al., Science 339, 798 (2013).

[11] M. A. Broome, A. Fedrizzi, S. Rahimi-Keshari, J. Dove, S. Aaronson, T. C. Ralph, and A. G. White, Science 339, 794 (2013).

[12] M. Tillmann, B. Dakić, R. Heilmann, S. Nolte, A. Szameit, and P. Walther, Nature Photon. 7, 540 (2013).

[13] A. Crespi, R. Osellame, R. Ramponi, D. J. Brod, E. F. Galvao, N. Spagnolo, C. Vitelli, E. Maiorino, P. Mataloni, and F. Sciarrino, Nature Photon. 7, 545 (2013).

[14] N. Spagnolo, C. Vitelli, M. Bentivegna, D. Brod, A. Crespi, F. Flamini, S. Giacomini, G. Milani, R. Ramponi, P. Mataloni, R. Osellame, E. F. Galvao, and F. Sciarrino, Nature Photon. 8, 615 (2014).
[15] P. Shadbolt, M. Verde, A. Peruzzo, A. Politi, A. Laing, M. Lobino, J. Matthews, M. Thompson, and J. O'Brien, Nature Photon. 6, 45 (2012).

[16] M. Reck, A. Zeilinger, H. J. Bernstein, and P. Bertani, Phys. Rev. Lett. 73, 58 (1994).

[17] S. Aaronson and A. Arkhipov, arXiv:1309.7460.

[18] M. C. Tichy, K. Mayer, A. Buchleitner, and K. Mølmer, Phys. Rev. Lett. 113, 020502 (2014).

[19] M. Walschaers, J. Kuipers, J.-D. Urbina, K. Mayer, M. C. Tichy, K. Richter, and A. Buchleitner, arXiv:1410.8547.

[20] Y. L. Lim and A. Beige, New J. Phys. 7, 155 (2005).

[21] M. C. Tichy, M. Tiersch, F. Mintert, and A. Buchleitner, New J. Phys. 14, 093015 (2012).

[22] G.-S. Cheon and I. M. Wanless, Linear Algebra and its Applications 403, 314 (2005).

[23] R. Simion and F. W. Schmidt, Discrete Mathematics 46, 107 (1983).

[24] Note that, for large $a$, one has

$$
\begin{aligned}
\frac{a !}{(a+b) !} & =\frac{1}{(a+b)(a+b-1) \ldots(a+1)} \\
& =\frac{1}{a^{b}\left(1+\frac{b}{a}\right)\left(1+\frac{b-1}{a}\right) \ldots\left(1+\frac{1}{a}\right)} \asymp \frac{1}{a^{b}} .
\end{aligned}
$$

[25] In fact, each sign inversion of a column inverts the sign of the permanent, but this is performed an even number of times.

[26] Incidentally, we note that this is also a partial answer to an open problem regarding permanents [22], i.e., the question whether the permanent of a Hadamard matrix could in general vanish.

[27] Y. Omar, N. Paunković, L. Sheridan, and S. Bose, Phys. Rev. A 74, 042304 (2006).

[28] J. Matthews, K. Poulios, J. Meinecke, A. Politi, A. Peruzzo, N. Ismail, K. Worhoff, M. Thompson, and J. O'Brien, Scientific Reports 3, 1539 (2013).

[29] A. P. Lund, A. Laing, S. Rahimi-Keshari, T. Rudolph, J. L. O'Brien, and T. C. Ralph, Phys. Rev. Lett. 113, 100502 (2014). 